\title{
Hysteresis phenomena at ultrathin lubricant film melting in the case of first-order phase transition
}

\author{
A.V. Khomenko*, I.A. Lyashenko \\ Sumy State University, Rimskii-Korsakov St. 2, 40007 Sumy, Ukraine \\ Received 26 September 2006; received in revised form 21 December 2006; accepted 5 February 2007 \\ Available online 9 February 2007 \\ Communicated by R. Wu
}

\begin{abstract}
Within the framework of Lorentz model for description of viscoelastic medium the influence of deformational defect of the shear modulus is studied on melting of ultrathin lubricant film confined between the atomically flat solid surfaces. The possibility of jump-like and continuous melting is shown. Three modes of lubricant behavior are found, which correspond to the zero shear stress, the Hooke section of loading diagram, and the domain of plastic flow. Transition between these modes can take place according to mechanisms of first-order and second-order phase transformations. Hysteresis of dependencies of stationary stresses on strain and friction surfaces temperature is described. Phase kinetics of the system is investigated. It is shown that ratio of the relaxation times for the studied quantities influences qualitatively on the character of the stationary mode setting.

(C) 2007 Elsevier B.V. All rights reserved.
\end{abstract}

PACS: 64.60.-i; 62.20.Fe; 62.20.Qp; 68.60.-p

Keywords: Boundary friction; Viscoelastic medium; Shear stress and strain; Stick-slip friction

\section{Introduction}

Last years the problems of sliding friction of smooth solid surfaces with thin lubricant film between them attract considerable attention [1]. The described boundary mode of friction is realized in the case of ultrathin lubricant films with thickness less than four diameters of molecules at smooth surfaces or asperities, high loads and low shear rates. It is characterized by the following changes of static (equilibrium) and dynamic properties of lubricant-simple unstructured Newtonian liquid (see Ref. [2] and the literature cited therein):

- non-fluid-like (non-Newtonian) properties: transition between liquid and solid phases, appearance of new liquidcrystalline states, epitaxially induced long-range ordering;

\footnotetext{
* Corresponding author.

E-mail addresses: khom@phe.sumdu.edu.ua (A.V. Khomenko), nabla@cable-tv.sumy.ua (I.A. Lyashenko).
}

- tribological properties: absence of flow until yield point or critical shear stress reached, solid-like behavior of lubricant characterized by defect diffusion and dislocation motion, shear and jump-like melting, boundary lubrication.

Experiments were carried out with mica, silica, metal oxide, and surfactant monolayer surfaces with organic liquids and aqueous solutions between them. It has been shown that there were transformations between the different types of dynamic phases during sliding [2]. They manifest themselves in appearance of intermittent (stick-slip) friction [3,4], which is characterized by periodic transitions between two or more dynamic states during the stationary sliding. The stick-slip friction is the major reason for destruction and wear of rubbing parts. Thus, molecularly thin lubricant films undergo more than one type of transition that results to existence of different types of stick-slip motion.

In the previous work [5] the approach is developed according to which the transition of ultrathin lubricant film from solidlike to liquid-like state takes place as a result of thermodynamic 
and shear melting. The combined analytical description of these processes is carried out due to self-organization of the fields of shear stresses and strain, and temperature of lubricant film also. Additive noises of the indicated quantities, different types of temperature dependence of viscosity, and correlated fluctuations of temperature are taken into account [6-8].

However, there a question was not considered about the reasons for the jump-like melting and hysteresis, which were observed in the experiments $[9,10]$. The proposed work is devoted to finding the realization conditions of these features taking into account the deformational defect of the shear modulus. Our approach is based on the Lorentz model for approximation of viscoelastic medium [5] in the case when the first-order phase transition is realized. Three stationary modes are found-two solid-like, corresponding to dry friction, and one liquid-like, meeting the sliding. The phase portraits of the system are built. The various interrupted modes of friction depending on initial conditions until setting of equilibrium can be realized. It is also shown that the hysteresis manifests itself in phase portraits in the case, when relaxation time of strain is much longer than corresponding times for stresses and temperature.

\section{Basic equations}

In the previous work [5] on the basis of rheological description of viscoelastic medium, possessing heat conductivity, the system of kinetic equations is obtained, which determine the mutually coordinated behavior of the shear stresses $\sigma$ and strain $\varepsilon$, and the temperature $T$ in ultrathin lubricant film during the process of friction between atomically flat mica surfaces. Let us write down these equations using the measure units:

$\sigma_{s}=\left(\frac{\rho c_{v} \eta_{0} T_{c}}{\tau_{T}}\right)^{1 / 2}$

$\varepsilon_{s}=\frac{\sigma_{s}}{G_{0}} \equiv\left(\frac{\tau_{\varepsilon}}{\tau_{T}}\right)^{1 / 2}\left(\frac{\rho c_{v} T_{c} \tau_{\varepsilon}}{\eta_{0}}\right)^{1 / 2}, \quad T_{c}$

for variables $\sigma, \varepsilon, T$, respectively, where $\rho$ is the mass density, $c_{v}$ is the specific heat capacity, $T_{c}$ is the characteristic temperature, $\eta_{0} \equiv \eta\left(T=2 T_{c}\right)$ is the characteristic value of shear viscosity $\eta, \tau_{T} \equiv \rho l^{2} c_{v} / \kappa, l$, and $\kappa$ are the time, the scale, and the coefficient of heat conductivity, correspondingly, $\tau_{\varepsilon}$ is the relaxation time of strain, $G_{0} \equiv \eta_{0} / \tau_{\varepsilon}$ :

$\tau_{\sigma} \dot{\sigma}=-\sigma+g(\sigma) \varepsilon$,

$\tau_{\varepsilon} \dot{\varepsilon}=-\varepsilon+(T-1) \sigma$,

$\tau_{T} \dot{T}=\left(T_{e}-T\right)-\sigma \varepsilon+\sigma^{2}$.

Here the relaxation time of stress $\tau_{\sigma}$, the temperature $T_{e}$ of atomically flat mica surfaces of friction, and the function $g(\sigma) \equiv G(\sigma) / G_{0}$ are introduced, where $G(\sigma)$ is the shear modulus of lubricant depending on the stress value:

$G(\sigma)=\Theta+\frac{G-\Theta}{1+\left(\sigma / \sigma_{p}\right)^{\beta}}, \quad \beta=$ const $>0$.

At $g(\sigma)=G / G_{0} \equiv$ const, Eq. (2) is reduced to the Maxwelltype equation for description of viscoelastic medium by substituting $\partial \varepsilon / \partial t$ for $\varepsilon / \tau_{\sigma}$. The Maxwell equation supposes the use of the idealized Genki model. For the dependence of stress on strain $\sigma(\varepsilon)$ this model is represented by the Hooke law $\sigma=G \varepsilon$ at $\varepsilon<\varepsilon_{m}$ and constant $\sigma_{m}=G \varepsilon_{m}$ at $\varepsilon \geqslant \varepsilon_{m}\left(\sigma_{m}, \varepsilon_{m}\right.$ are the maximal values of elastic shear stress and strain for the Hooke section, $\sigma>\sigma_{m}$ results in the viscous flow with deformation rate $\left.\dot{\varepsilon}=\left(\sigma-\sigma_{m}\right) / \eta\right)$. Actually, the curve of dependence $\sigma(\varepsilon)$ is characterized by two regions: first one, Hookean, has the large slope determined by the shear modulus $G$, and it is followed by the more gently sloping section of plastic deformation whose tilt is defined by the hardening factor $\Theta<G$. Obviously, the above picture means that the shear modulus depends on the value of stresses. In order to take into account this circumstance we use the simplest approximation (5), which describes the above represented transition of elastic deformation mode to the plastic one. It takes place at the characteristic values of shear stress $\sigma_{p}$ and strain $\varepsilon_{p}$. It is worth noting that at description of structural phase transitions of liquid-like lubricant the third-order invariants are present, which breaks the parity of the dependence of the synergetic potential $V$ on the stress $\sigma$. Therefore in approximation (5) the linear term was used $\sigma / \sigma_{p}$ $(\beta=1)$, instead of square one $\left(\sigma / \sigma_{p}\right)^{2}(\beta=2)$ [11], and dependence $V(\sigma)$ was not already even [5]. Consequently, the odd values of $\beta$ correspond to the sliding friction experiment at which the motion of stage in negative and positive directions is not equivalent energetically.

Expression (3) has the form of the corresponding KelvinVoigt equation [5,12], which takes into account the dependence of the shear viscosity on the dimensionless temperature

$\eta=\frac{\eta_{0}}{T-1}$.

Note that jointly Eqs. (2) and (3) represent the new rheological model, since they are reduced to the second-order differential equation with respect to the stress $\sigma$ or the strain $\varepsilon$. Eq. (4) is the expression for heat conductivity, which describes the transmission of heat from the friction surfaces to lubricant film, the effect of dissipative heating of viscous liquid flowing under the action of stress, and the reversible mechanic-and-caloric effect in linear approximation. Eqs. (2)-(4) formally coincide with the synergetic Lorentz system $[11,13]$, in which the shear stress acts as the order parameter, the conjugate field is reduced to the shear strain, and the temperature represents the control parameter. As is known this system is used in describing both phase thermodynamic and kinetic transformations. It is necessary also to note that rheological properties of lubricant film are investigated experimentally that makes it possible to build a phase diagram [2].

Dependence (5) describes hysteresis at melting of thin layer of lubricant only in coordinates $T_{e}-\sigma$ during realization of first-order phase transition [5]. Thus, deformation curve $\sigma(\varepsilon)$ is monotonous and in the case of second-order phase transition it allows us to represent only continuous transition. However, experimental data testify that melting of molecularly thin lubricant film has jump-like character [2], although it may take place according to the mechanism of second-order phase transition. As is shown below the description of the indicated feature is achieved replacing the dependence $g(\sigma)$ in (2) by $g(\varepsilon) \equiv$ 
$G(\varepsilon) / G_{0}$, where

$G(\varepsilon)=\Theta+\frac{G-\Theta}{1+\left(\varepsilon / \varepsilon_{p}\right)^{\beta}}$.

At this time the value of parameter $\beta>0$, determining the potential character, plays a key role also.

In the work [5] the melting of ultrathin lubricant film during the process of friction between the atomically flat surfaces of mica is represented as a result of spontaneous appearance of shear stresses resulting in plastic flow. This is caused by heating of friction surfaces above the critical value $T_{c 0}=1+G_{0} / G$. The initial reason for self-organization process is the positive feedback of $T$ and $\sigma$ on $\varepsilon$ [see (3)], conditioned by temperature dependence of shear viscosity leading to its divergence. On the other hand, the negative feedback of $\sigma$ and $\varepsilon$ on $T$ in (4) has an important role since it provides the system stability.

In accordance with such approach the lubricant represents a strongly viscous liquid that can behave similar to the solid. It has large effective viscosity and is still characterized by the yield stress [2,12]. The solid-like state of lubricant corresponds to the shear stress $\sigma=0$, since in this case Eq. (2) falls out of consideration. ${ }^{1}$ Eq. (3), containing the viscous stresses, is reduced to the Debye law representing the rapid relaxation of shear strain during microscopic time $\tau_{\varepsilon} \approx a / c \sim 10^{-12} \mathrm{~s}$, where $a \sim 1 \mathrm{~nm}$ is the lattice constant or the intermolecular distance and $c \sim 10^{3} \mathrm{~m} / \mathrm{s}$ is the sound velocity. Thus, equation of heat conductivity (4) assumes the form of simplest expression for temperature relaxation that does not contain the terms representing dissipative heating and mechanic-and-caloric effect of viscous liquid.

Eq. (3) describes the plastic flow of lubricant with velocity $V=h \partial \varepsilon / \partial t$ due to action of appearing viscous shear stress $(h$ is the film thickness). Particularly, in the case of surface force apparatus [10] in Eqs. (2)-(4) the effective strain amplitude $\varepsilon=$ $x_{\max } / h$ is defined as the ratio of the deformation (deflection) amplitude $x_{\max }$ to the $h$ value. The effective shear rate $\dot{\varepsilon}=\varepsilon \omega=$ $V / h=\varepsilon / \tau_{\sigma}$ is the product of the strain $\varepsilon$ and the oscillation frequency $\omega$. In experiments where the driving force is found we have the following expressions for viscous friction force and shear stress [14]:

$F_{v}=\eta_{\mathrm{eff}} V A / h, \quad \sigma_{v}=F_{v} / A$,

where $\eta_{\text {eff }}$ is the effective viscosity, $A$ is the area of contact. According to (8) the driving velocity is $V=\sigma_{v} h / \eta_{\text {eff. In this case }}$ the effective viscosity does not coincide with the real viscosity and can be found only in experiment.

It is shown [15] that the plastic flow of lubricant layer is realized at presence of elastic stress. The action of shear stress results to reducing of shear modulus of lubricating material [16]. Consequently, the total friction force decreases with increasing velocity at the contact $V$ because the latter leads to the growth of the shear stress according to the Maxwell stressstrain $\varepsilon$ relationship: $\partial \sigma / \partial t=-\sigma / \tau_{\sigma}+G \partial \varepsilon / \partial t$.

\footnotetext{
1 It will be shown further that non-zero stresses interval can meet the solidlike state of lubricant also.
}

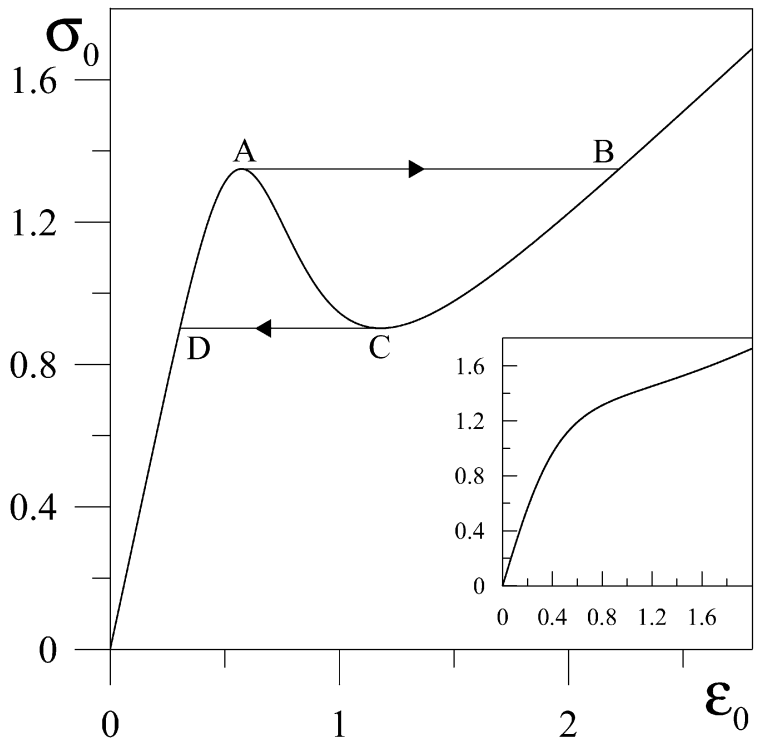

Fig. 1. The dependence of the stationary values of the shear stresses $\sigma_{0}$ on the strain $\varepsilon_{0}$ (9) at $\theta=0.2, g_{\theta}=0.6, \alpha=0.7, \beta=5.0$ (in the insert $\beta=2.0$ ).

Moreover, in accordance with Ref. [4] in the absence of shear deformations the temperature mean-square displacement of molecules (atoms) is defined by equality $\left\langle u^{2}\right\rangle=T / G a$. The average shear displacement is found from the relationship $\left\langle u^{2}\right\rangle=\sigma^{2} a^{2} / G^{2}$. The total mean-square displacement represents the sum of these expressions provided that the thermal fluctuations and the stress are independent. Above implies that the melting of lubricant is induced both by heating and under the effect of stress generated by solid surfaces in the course of friction [4]. It is assumed that the film becomes more liquid-like and the friction force decreases with the temperature growth due to activation energy barrier decreasing to molecular hops.

\section{Hysteresis behavior}

Let us consider the stationary state, at which the derivative $\dot{\sigma}=0$ in Eq. (2), and value $\sigma$ does not change in the lubricant. Then, the equation is obtained similar to the Hooke law:

$\sigma=g(\varepsilon) \varepsilon, \quad g(\varepsilon)=g_{\theta}\left(1+\frac{\theta^{-1}-1}{1+(\varepsilon / \alpha)^{\beta}}\right)$,

where parameter $\theta=\Theta / G<1$, determining the ratio of the tilts for the deformation curve on the plastic and the Hookean sections, coefficients $g_{\theta}=\Theta / G_{0}<1$ and $\alpha=\varepsilon_{p} / \varepsilon_{s}$ are introduced.

Dependence (9) at fixed $\alpha, g_{\theta}$, and $\theta$ is depictured in Fig. 1. In the case of surface force apparatus [10] it presents the dependence of the responding total shear stress in lubricant $\sigma=\sigma_{\mathrm{el}}+\sigma_{v}$ on the deflection amplitude $x_{\max }$, where $\sigma_{\mathrm{el}}$ and $\sigma_{v}=\eta_{\mathrm{eff}} V / h$ are the elastic and the viscous parts, respectively. It is apparent from here that two situations can be realized: at small $\beta$ the curve $\sigma(\varepsilon)$ increases monotonically (insert in the figure), and at

$\beta>\frac{1+\sqrt{\theta}}{1-\sqrt{\theta}}$ 


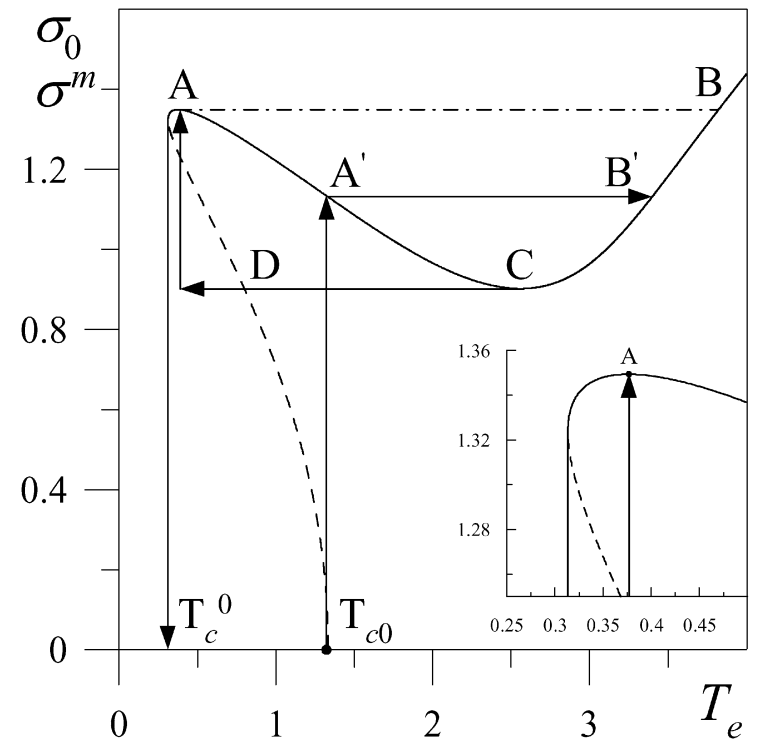

(a)

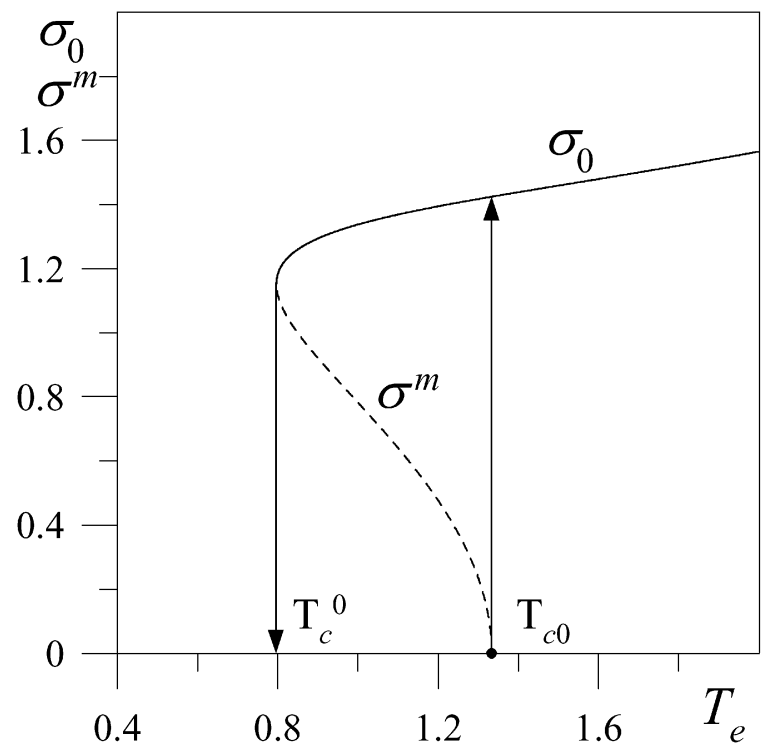

(b)

Fig. 2. The dependence of the stationary values of the shear stresses $\sigma_{0}, \sigma^{m}$ on the temperature of friction surfaces $T_{e}$ at parameters of Fig. 1 (Fig. $2 \mathrm{~b}$ corresponds to the parameters of insert in Fig. 1).

it becomes non-monotonous. In the first case in accordance with the figure the continuous melting of lubricant takes place, in the second one-lubricant melts abruptly at the increase of stresses to the point $A$, and here the transition to point $B$ occurs. At the further growth of the stresses the strain increases monotonically, and lubricant is liquid-like. If stress is decreased now to the point $C$ the lubricant conserves liquid-like structure, and then it abruptly becomes solid during transition at point $D$. At the subsequent decrease of stresses lubricant is solid-like. Similar transformations are represented as the first-order phase transitions [17], but between the states, which are not the pure thermodynamic phases. In describing these transformations the concept of shear melting is used [4]. Note that such hysteresis behavior was observed in the experiments $[9,10,15]$.

Using (9), it is possible to find the abscissas of transition points $A$ and $C$ :

$$
\begin{aligned}
\varepsilon_{A, C} & =2^{-1 / \beta} \alpha\left[b(\beta-1)-2 \mp b \sqrt{(1-\beta)^{2}-4 \beta / b}\right]^{1 / \beta}, \\
b & =\theta^{-1}-1,
\end{aligned}
$$

where the sign ' - ' corresponds to the point $A$, and sign ' + 'to the point $C$. From Eq. (11) it is seen that the length of jump at melting increases with growth $\alpha$, and the difference $\varepsilon_{A}-$ $\varepsilon_{C} \rightarrow 0$ with the increase of $\beta$. Thus, at large $\beta$ (small $\alpha$ ) melting and solidification occur at the same value of strain $\left(\varepsilon_{A} \approx \varepsilon_{C}\right)$ practically, but at different values of stresses $\sigma$. As well as in Refs. [5-8], we will accept as the order parameter the shear stress $\sigma$ : at $\sigma>\sigma_{A}$ lubricant is liquid-like, and if $\sigma<\sigma_{C}$ it is solid-like. In the intermediate region $\sigma_{C}<\sigma<\sigma_{A}$ the state of lubricant is unstable, since it may exist in both phases.

For further consideration it is necessary to write down expression for synergetic potential $V(\sigma)$. Within the framework of adiabatic approximation $\tau_{\varepsilon}, \tau_{T} \ll \tau_{\sigma}[5,6]$ it is possible to set $\tau_{\varepsilon} \dot{\varepsilon} \approx 0, \tau_{T} \dot{T} \approx 0$, and Eqs. (3), (4) give

$\varepsilon=\sigma-\left(2-T_{e}\right) \frac{\sigma}{1+\sigma^{2}}$,

$T=T_{e}+\left(2-T_{e}\right) \frac{\sigma^{2}}{1+\sigma^{2}}$.

After substitution of expression (12) in (2) we obtain the Landau-Khalatnikov equation:

$\tau_{\sigma} \dot{\sigma}=-\frac{\partial V}{\partial \sigma}$,

where the synergetic potential is defined by equality

$$
\begin{aligned}
V= & \frac{\sigma^{2}}{2}-g_{\theta} \int_{0}^{\sigma}\left[\sigma-\left(2-T_{e}\right) \frac{\sigma}{1+\sigma^{2}}\right] \\
& \times\left[1+\frac{\theta^{-1}-1}{1+\left(\sigma / \alpha-\sigma\left(2-T_{e}\right) /\left(\alpha+\alpha \sigma^{2}\right)\right)^{\beta}}\right] d \sigma .
\end{aligned}
$$

The dependence of the stationary shear stresses $\sigma_{0}, \sigma^{m}$ on the temperature of friction surfaces $T_{e}$ is presented in Fig. 2 at parameters of Fig. 1 (Fig. 2b meets the insert in Fig. 1). Apparently that it is non-monotonous, and in the interval $T_{c}^{0}<$ $T_{e}<T_{c 0}$ the two-valued section is realized inherent to firstorder phase transitions. The dashed curve corresponds to the unstable stationary values of stresses $\sigma^{m}$, the solid curve-to the stable $\sigma_{0}$. It is worth noting that $\sigma^{m}\left(T_{e}\right)$ meets the Hookean section of dependence $\sigma_{0}\left(\varepsilon_{0}\right)$.

Note that in the region $T_{c A}<T_{e}<T_{c C}$ the potential (15) does not give correct result, since here the model has the unstable solution, describing the decrease of stresses with growth of strain, and this does not take into account hysteresis. In finding the form of potential in the indicated region we replace (2) by equation

$\tau_{\sigma} \dot{\sigma}=-\sigma+\delta$, 


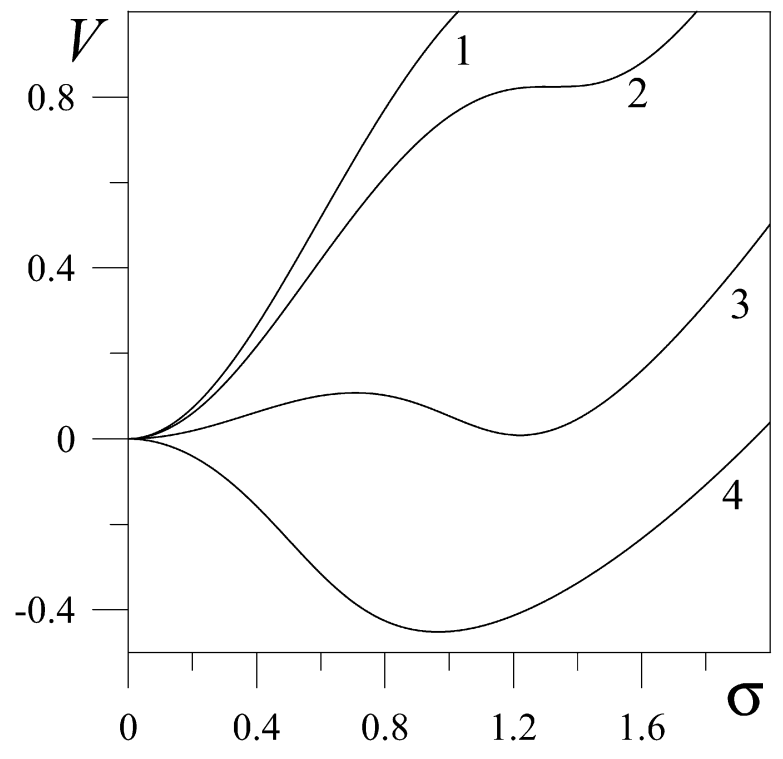

Fig. 3. The dependence of the synergetic potential $V$ (15) on the shear stresses $\sigma$ at parameters of Fig. 2a, curves $1-4$ correspond to the values of temperature $T_{e}=0.1,0.3125,1.0,2.0$, respectively.

where $\delta$ is the value of the conserved stresses. In the corresponding Landau-Khalatnikov equation (14) the potential is fixed by expression

$V^{\prime}=\frac{\sigma^{2}}{2}-\delta \sigma$.

It is seen that $V^{\prime}$ depends only on $\sigma$, i.e., in the situation, where stress is conserved at the change of temperature, the value $V^{\prime}$ remains constant.

The dependence of the potential (15) on the stress value at the fixed temperatures of friction surfaces is presented in Fig. 3 corresponding to the parameters of Fig. 2a. The hysteresis, shown in Fig. 1, is realized in this situation. We will consider this case in more detail.

Below than the critical value $T_{c}^{0}$ stresses are absent in lubricant $\left(\sigma_{0}=0\right)$. The form of potential, shown by curve 1 in Fig. 3, corresponds to this interval of temperatures. Here one zero minimum is realized at $\sigma_{0}=0$, thus lubricant is solidlike. At point $T_{e}=T_{c}^{0}$ a plateau appears on the dependence $V(\sigma)$ (curve 2). With the further increase of temperature in a region $T_{c}^{0}<T_{e}<T_{c 0}$ the potential has the form shown by curve 3 . Here the potential barrier appears, which separates the zero and the non-zero minimums of potential. In connection with it the system cannot come in the stable state $\sigma_{0} \neq 0$, and the zero value of shear stress $\sigma$ is realized. The dashed curve in Fig. 2a corresponds to the maximum of potential, the solid curve-to its non-zero minimum. As is apparent from figure at $T_{e}=T_{c 0}$ the jump-like increase of value $\sigma$ takes place, and the system turns to the section of dependence $\sigma_{0}\left(T_{e}\right)$ (point $A^{\prime}$ ). This transition is caused by that at $T_{e}=T_{c 0}$ the maximum $V(\sigma)$ disappears, and with subsequent growth of $T_{e}$ the one non-zero minimum of potential is realized (curve 4 in Fig. 3 ). The expression for the critical temperature $T_{c 0}$ is obtained from the condition $\partial V / \partial \sigma=0$, where $V$ is the synergetic poten- tial (15):

$T_{c 0}=1+\theta / g_{\theta} \equiv 1+G_{0} / G$.

However, section $A C$ is unstable, because here the stress is decreased at growth of strain $(A, B, C$, and $D$ meet the similar points in Fig. 1). In this connection further the system goes on the way $A^{\prime}-B^{\prime}$ (this transition is already described by potential $V^{\prime}(17)$, since stress is conserved), and passes to the plastic flow section, which corresponds to the liquid-like structure of lubricant. With the subsequent increase of temperature $T_{e}$ the stationary value of stresses $\sigma_{0}$ grows, and lubricant becomes less viscous, here $V(\sigma)$ has the form shown by curve 4 . If now the temperature of friction surfaces is decreased, the lubricant is liquid-like till the value $T_{c}$, further the stress is conserved to the critical $T_{e}=T_{c A}$ (corresponding to the point $A$ ). ${ }^{2}$ As is seen from the insert in the picture at $T_{e}=T_{c A}$ the system passes to the point $A$, because here the dependence $\sigma_{0}\left(T_{e}\right)$ becomes stable. At this transition system of somehow "jump over" the potential barrier (dashed curve of the dependence). It is related to that before the jump the system is described by other potential $V^{\prime}$, which is characterized by absence of barrier. Here the solidification of lubricant occurs, because transition takes place on the stable part of Hooke section (according to Fig. 1 temperature $T_{e}<T_{c A}$ corresponds to the Hooke domain). Now, the system is described by potential $V(\sigma)(15)$ with the barrier shown by curve 3 in Fig. 3. With the further decrease of the temperature of friction surfaces at the point $T_{e}=T_{c}^{0}$ stresses decrease abruptly to the zero, since the barrier disappears and there is one zero minimum of $V(\sigma)$. This situation corresponds to the solidlike state of lubricant also, but with the zero value of stress. It is supposed that the solid-like states at $\sigma_{0}=0$ and $\sigma_{0} \neq 0$ differ by structure, because of transition between them takes place according to the mechanism of the first-order phase transition. Thus, the solid-like structure of lubricant at a temperature below $T_{c}^{0}$ is similar to the solid state, and the solid-like structure above indicated temperature has the signs of the liquid state, but lubricant behaves as a solid-like on the whole. The different solid-like states correspond to the different modes of friction in the studied system [18]. At further transition to the liquid mode of friction the viscosity of lubricant (6) decreases, and it flows.

At described transitions the stationary values of stresses are conserved in intervals $T_{c A^{\prime}}<T_{e}<T_{c B^{\prime}}$ and $T_{c A}<T_{e}<T_{c C}$. Obviously, that equality $\sigma_{0}=$ const is satisfied with the increase of temperature at $T_{c A^{\prime}}<T_{e}<T_{c B^{\prime}}$, since it is necessary to give some energy to lubricant for melting. Conservation of stresses in the region $T_{c A}<T_{e}<T_{c C}$ at decrease of the sheared surfaces temperature takes place, because for transition of lubricant in the solid-like state it has to return energy. It is worth noting that at the conservation of stresses between solid-like and liquid-like phases of lubricant, it is in the intermediate state with differing from them structure.

\footnotetext{
2 In general case the system has to move along the curve $C A$, but it is unstable. Therefore it is necessary to introduce the described hysteresis, and to take into account that at this transition the system is described by potential $V^{\prime}(\sigma)$ (17).
} 


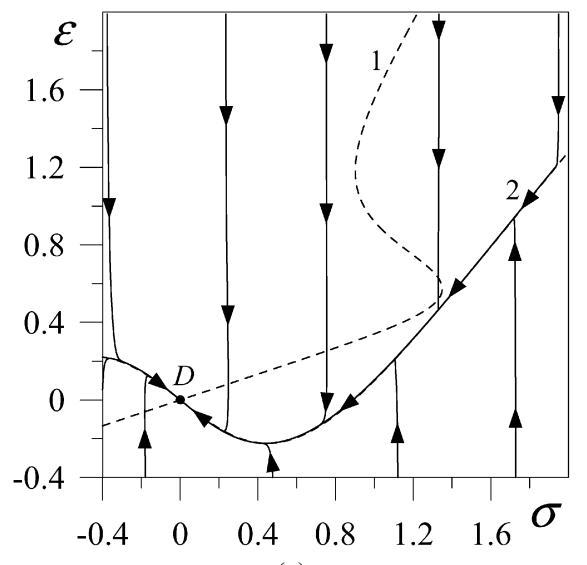

(a)

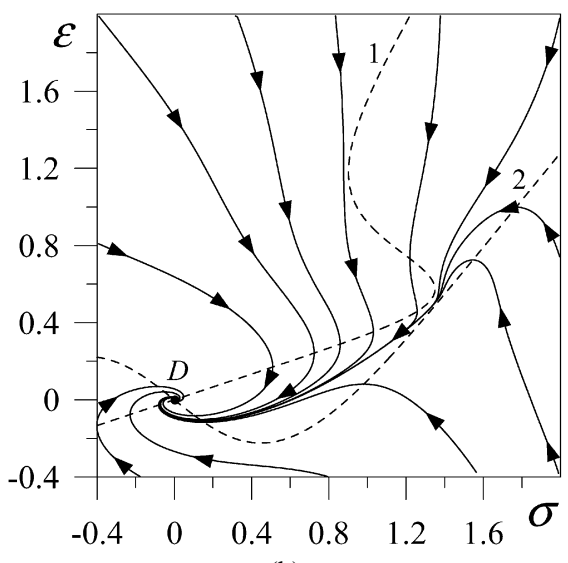

(b)

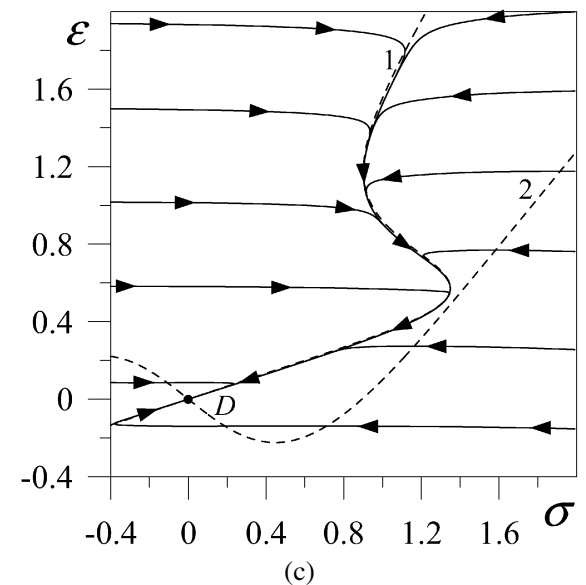

(c)

Fig. 4. The phase portraits at parameters of Fig. 2a and $T_{e}=0.2$ : (a) $\tau_{T} \ll \tau_{\varepsilon}=0.01 \tau_{\sigma}$; (b) $\tau_{T} \ll \tau_{\varepsilon}=\tau_{\sigma}$; (c) $\tau_{T} \ll \tau_{\varepsilon}=100 \tau_{\sigma}$.

The situation, which is given by the insert to Fig. 1, is shown in Fig. $2 \mathrm{~b}$ when hysteresis is not realized in coordinates $\sigma_{0}\left(\varepsilon_{0}\right)$. This corresponds to the more simple case described in work [5]. Below the value $T_{c 0}$ we have the solid-like state of lubricant $\left(\sigma_{0}=0\right)$, at $T_{e}=T_{c 0}$ it melts at transition to the plastic flow section, and with the subsequent increase of $T_{e}$ it becomes more liquid. Then, with the decrease of temperature to the value $T_{c}^{0}$ lubricant is liquid-like $\left(\sigma_{0} \neq 0\right)$, at $T_{e}=T_{c}^{0}$ it solidifies abruptly $\left(\sigma_{0}=0\right)$.

The distinctive feature of such behavior is that whole Hooke domain is unstable, since it meets the maximum of potential. There is only one type of solid-like state of lubricant with the zero value of stress.

Depending on the parameters of the system here the secondorder phase transition can be observed, when the temperature $T_{c 0}$ is moved on the left-hand side from $T_{c}^{0}$. Indeed, the unstable stationary values of stresses $\sigma^{m}$ are not realized, and hysteresis disappears, which is characterized by a presence of $T_{c}^{0}$. In this case the potential, shown by curve 1 in Fig. 3, corresponds to the solid-like structure of lubricant, which is transformed to the form represented by curve 4 with the increase of temperature. Thus, the continuous transformation takes place, since the potential barrier is absent. Here it is also possible to select two situations-when hysteresis of $\sigma_{0}\left(\varepsilon_{0}\right)$ dependence is observed, and without it.

\section{Phase kinetics}

In accordance with the experimental data for organic lubricating materials $[2,4,6]$ the relaxation time of stresses at normal pressure is $\tau_{\sigma} \sim 10^{-10} \mathrm{~s}$. Since ultrathin lubricant film has less than four molecular layers, temperature relaxes to the value $T_{e}$ during time satisfying the condition:

$\tau_{T} \ll \tau_{\sigma}, \tau_{\varepsilon}$.

According to this we assume in Eq. (4) $\tau_{T} \dot{T} \approx 0$. Also for convenience the time is measured in units of $\tau_{\sigma}$. As a result, we obtain the two-parameter system in the form:

$\dot{\sigma}=-\sigma+g(\varepsilon) \varepsilon$, $\tau \dot{\varepsilon}=-\varepsilon+\left(T_{e}-1-\sigma \varepsilon+\sigma^{2}\right) \sigma$,

where $\tau \equiv \tau_{\varepsilon} / \tau_{\sigma}$. The corresponding phase portraits are presented in Figs. 4-6 at the parameters of Fig. 2a for different $T_{e}$.

The phase portraits describing the behavior of lubricant in the solid-like state $\left(T_{e}<T_{c}^{0}\right)$ are shown in Fig. 4 for different ratios of relaxation times.

Particularly, Fig. 4a corresponds to the value $\tau=0.01$. The isoclines obtained at equating to zero of derivatives in Eqs. (20) and (21) are shown by dashed curves 1 and 2, respectively. Thus, curve 1 meets the system parameters at which stresses do not change, and line 2 corresponds to the case of strain conservation. These lines intersect at the origin of coordinates forming the unique stationary point $D$ that is a node. It is apparent that phase trajectories converge to the node $D$, i.e., stresses relax to the zero value. Thus, at motion on a phase plane at the arbitrary initial conditions there are two stages: on the first one, the instantaneous relaxation of the system takes place to the line near to isocline 2, on the second one-the slow motion on the indicated curve. At the first stage the stresses are conserved that reminds the transition between the friction modes described in Section 3. Apparently, on the final stage of system relaxation to the stationary point $D$ the values of stress and strain go out in the negative domain. This can be interpreted as reversible motion at which the top wall of friction moves in reverse direction. Thus, stresses change the sign in opposite one, because of the direction of motion changes, and strain becomes negative also. On the other hand, it is possible to neglect the negative region, considering it as has no physical meaning. We can assume that at achievement by strain the zero value the system abruptly goes to the origin of coordinates, and the equilibrium sets in.

The phase portrait shown in Fig. $4 \mathrm{~b}$ is built for the case when relaxation times of stresses and strain coincide $(\tau=1)$. It is also characterized by the singular point $D$, representing the node around which the weakly pronounced oscillations of short duration are realized now till setting of equilibrium. Here the cases are possible when stress $\sigma$ at first increases, and then decreases, and reverse situation. It means that to that moment, when the system will come to the equilibrium (the origin of the coordinates), the stick-slip motion is possible. For example, in accordance with phase trajectories, which begin at $\sigma=0$, the 


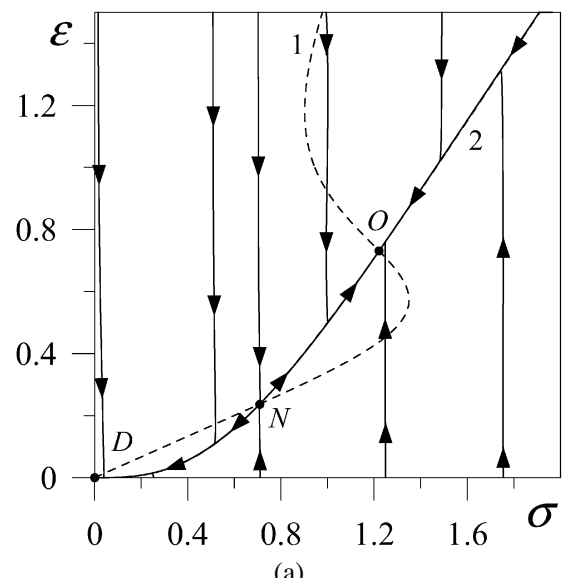

(a)

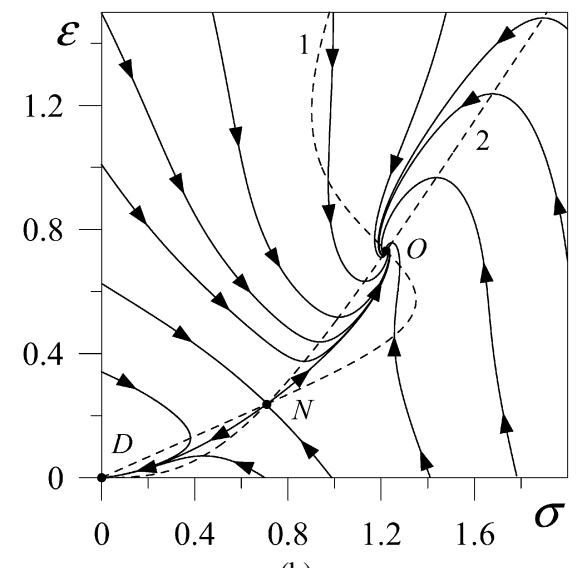

(b)

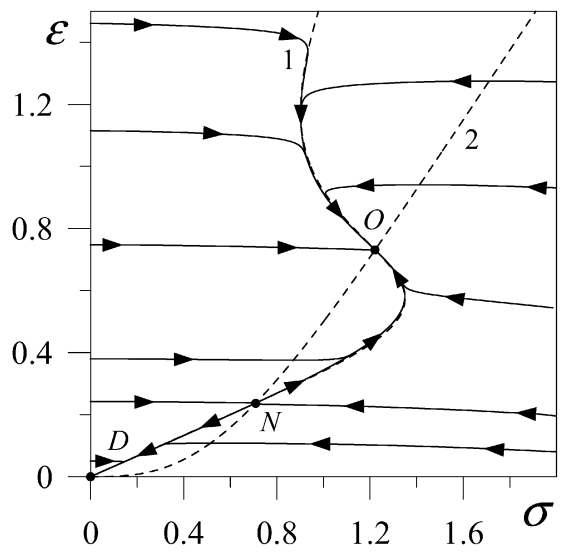

(c)

Fig. 5. The phase portraits at parameters of Fig. $2 \mathrm{a}$ and $T_{e}=1.0$ : (a) $\tau_{T} \ll \tau_{\varepsilon}=0.01 \tau_{\sigma}$; (b) $\tau_{T} \ll \tau_{\varepsilon}=\tau_{\sigma}$; (c) $\tau_{T} \ll \tau_{\varepsilon}=100 \tau_{\sigma}$.

lubricant is solid-like at first (stresses are equal to zero), then it begins to melt (stresses increase), and then it solidifies again (at setting of equilibrium). In order to avoid misunderstanding let us turn attention to the following circumstance. According to phase trajectories the cases are possible when the strain decreases with the growth of stresses. Above for the stationary values of stresses it was interpreted as instability resulting in hysteresis. Now, the rapid motion is realized. It allows us to assume that system is in the unstable state, and oscillation mode is realized in which the decrease of $\varepsilon$ is possible with the increase of $\sigma$.

Fig. 4c meets the case $\tau=100$. Here, as well as in Fig. 4a, the two stages are selected: the rapid relaxation to the line near to isocline 1 , and further the slow motion along it. At the first stage the strain changes weakly, and stresses decrease very rapidly, if their initial values are on the right-hand side from isocline 1 , or they increase at initial $\sigma$ on the left-hand side from it. At the second stage in the top part of phase portrait the configuration point moves along the plastic section, and in bottom one-along the Hooke domain. During the way of this point between the maximum and minimum of isocline 1 stresses increase with decrease of strain. The motion is slow here, and therefore the hysteresis has to be observed similar to described in Fig. 1. This feature is not shown in phase portrait, and its study requires the additional analysis, because there a lot of limiting cases can arise, which are not considered within the framework of the offered work.

The phase portraits in Fig. 5 are presented for the same parameters and ratios of relaxation times as in Fig. 4. But they are built for the temperature corresponding to the section of Fig. 2a, for which stable and unstable stationary values of shear stresses are realized $\left(T_{c}^{0}<T_{e}<T_{c 0}\right)$. In this case the system potential has the form shown by curve 3 in Fig. 3. As well as above, lines 1 and 2 are isoclines. The phase portraits are characterized by three singular points: by the node $D$ at the origin of coordinates, which describes the dry friction; by the saddle $N$ corresponding to the maximum of dependence $V(\sigma)$ (the unstable stationary point); by the node $O$ meeting the non-zero stationary stress, which corresponds to the unstable section $A C$ of dependence $\sigma_{0}\left(T_{e}\right)$ shown in Fig. 2a. These points are given by intersections of isoclines. Depending on the initial conditions the system may come as a result of relaxation both to the mode of stable dry friction (node $D$ ) and to the above described unstable section (node $O$ ).

At $\tau=0.01$ there is the picture shown in Fig. 5a. Here, as well as in Fig. 4a, phase trajectories rapidly converge to the line near to isocline 2 from any point of phase plane at conservation of stresses. Further the system relaxes to the nodes $D$, or $O$, and determined by these points the stationary modes of friction set in. The inclination of curve, along which motion occurs during the second stage, depends on initial conditions. So, the system relaxes to the point $O$ along the plastic section of isocline 2, to the point $D$-along its Hookean one. Note that in the course of time lubricant becomes more liquid, if $\sigma_{N}<\sigma_{i}<\sigma_{O}$, and, vice versa, more viscous at $\sigma_{i}>\sigma_{O}$, where $\sigma_{i}, \sigma_{N}$, and $\sigma_{O}$ are the initial and the stationary values of stresses. In these cases the system comes to the singular point $O$. At $\sigma_{i}<\sigma_{N}$ the lubricant solidifies during time, and dry friction is realized (point $D$ ).

As is obvious from Fig. 5b, for $\tau=1$ the different types of stick-slip motion are possible at setting of equilibrium values of stress and strain.

The phase portrait is shown in Fig. 5c for $\tau=100$, where, as well as above, there are two stages. Since the stationary point $O$ is on the unstable section, for the parameters of this figure the hysteresis is characteristic.

The phase portraits for the temperature domain of Fig. 2a, corresponding to the stable sliding friction $\left(T_{e}>T_{C}\right)$, are shown in Fig. 6. Here in the course of evolution the non-zero stationary value of shear stresses is set in $\sigma_{0} \neq 0$ meeting the minimum of synergetic potential $V(\sigma)$ (the maximum of distribution function of stresses $P(\sigma)$ over their value). The phase portraits are characterized by two singular points-by the saddle $D$ at the origin of coordinates and the node $O$ at the nonzero values of stress and strain, which are given by intersections of isoclines 1 and 2 .

At $\tau=0.01$ the situation is observed shown in Fig. 6a. Here, as well as above, phase trajectories converge rapidly to the line near to isocline 2 from any point of phase plane at conservation of stresses. Then, the system relaxes to the non-zero value $\sigma_{0} \neq 0$, as a result, the stationary sliding friction sets in. How- 


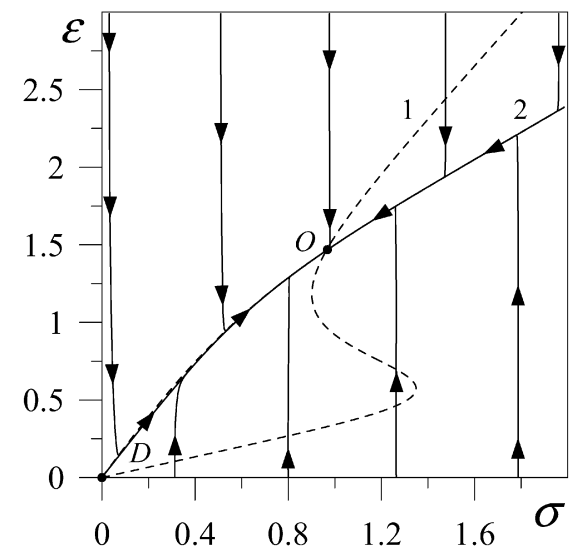

(a)

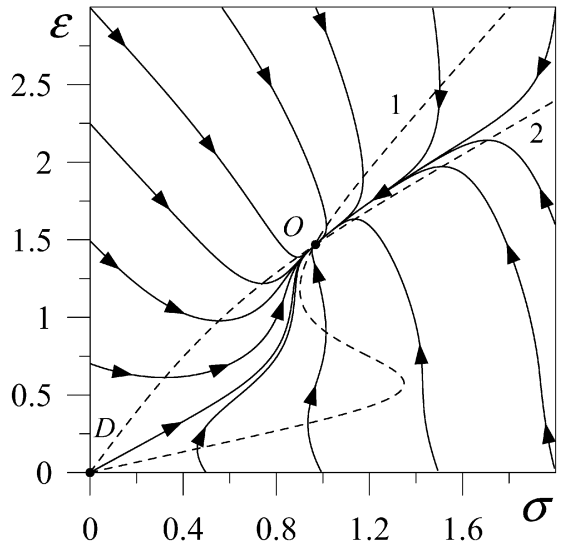

(b)

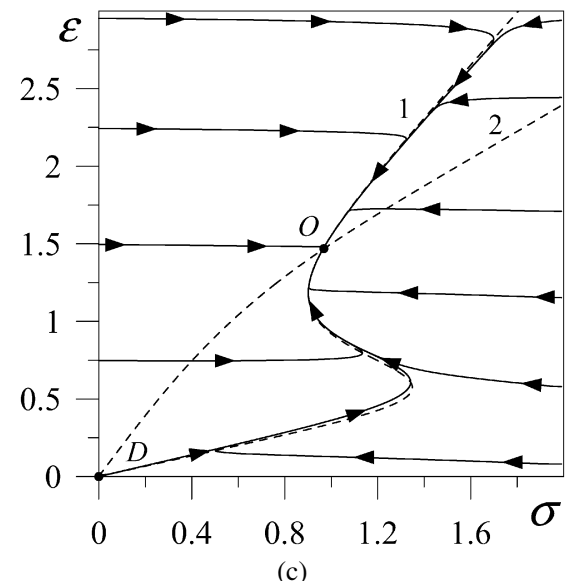

(c)

Fig. 6. The phase portraits at parameters of Fig. $2 \mathrm{a}$ and $T_{e}=3.0$ : (a) $\tau_{T} \ll \tau_{\varepsilon}=0.01 \tau_{\sigma}$; (b) $\tau_{T} \ll \tau_{\varepsilon}=\tau_{\sigma}$; (c) $\tau_{T} \ll \tau_{\varepsilon}=100 \tau_{\sigma}$.

ever, the curve, along which motion occurs during the second stage, corresponds to the plastic section of dependence $\sigma(\varepsilon)$, i.e., system is liquid-like always, except only the cases when initial value of stresses is near zero (the melting occurs). Note that in the course of time the lubricant becomes more liquid, if $\sigma_{i}<\sigma_{O}$, and, vice versa, more viscous at $\sigma_{i}>\sigma_{O}$.

As is apparent from Fig. 6b, for $\tau=1$ at setting of stationary sliding friction the different types of stick-slip motion are possible. However, at any initial conditions the system comes to the steady sliding friction.

The phase portrait is shown in Fig. $6 \mathrm{c}$ for $\tau=100$, where, as well as in Figs. $4 \mathrm{c}$ and $5 \mathrm{c}$ the two above described stages are seen. The stationary point $O$ is on the plastic flow region. The hysteresis is characteristic also for the parameters of this figure.

The difference of described in Figs. 4-6 situations is achieved due to the change of isocline 2 form with the variation of temperature $T_{e}$ of friction surfaces, while the form of isocline 1 does not depend on it. The value of $\beta$ influences substantially on the phase portraits, however, its variations do not change qualitatively the character of the system behavior. At $\tau \gg 1$ it is necessary to introduce hysteresis [19], because the slow motion of the system occurs near the isocline 1, and the condition of stationarity is valid in every time moment. The hysteresis is similar to described in Fig. 1, since the dependence, presented in this figure, represents isocline 1 shown in phase portraits.

\section{Conclusion}

The above consideration shows that the hysteresis, realized at melting of thin lubricant film according to the mechanism of first-order phase transition, can be described taking into account the deformational defect of the shear modulus. The basic feature of such behavior is that lubricant melts and solidifies at the different values of shear stress, which acts as the order parameter. The two solid-like states of lubricant and one liquidlike phase have been found, the transition between which takes place in accordance with indicated hysteresis. The phase kinetics is studied, and depending on initial conditions the different types of stick-slip motion are predicted. The elastic and thermal parameters of lubricant are defined, at which as a result of this motion the sliding, or the dry friction sets in. Particularly, it is shown that the ratio of shear stress and strain relaxation times, fixed by film thickness, defines different intermittent friction modes.

\section{Acknowledgements}

We express our gratitude to the organizers and participants of Working conference-seminar of Institute for Condensed Matter Physics of National Academy Sciences of Ukraine (2-3 of June, 2005, Lviv) within the framework V Ukrainian competition of young scientists in the field of statistical physics and theory of condensed medium for discussing and supporting of the work. We thank also Dr. A. Kiselev and Dr. S. Lukjanets for the discussion of work. The work was partly supported by a grant of the cabinet of Ukraine.

\section{References}

[1] B.N.J. Persson, Sliding friction, in: Physical Principles and Applications, Springer-Verlag, Berlin, 1998, p. 462.

[2] H. Yoshizawa, Y.-L. Chen, J. Israelachvili, J. Phys. Chem. 97 (1993) 4128; H. Yoshizawa, J. Israelachvili, J. Phys. Chem. 97 (1993) 11300.

[3] J.M. Carlson, A.A. Batista, Phys. Rev. E 53 (1996) 4153.

[4] I.S. Aranson, L.S. Tsimring, V.M. Vinokur, Phys. Rev. B 65 (2002) 125402.

[5] A.V. Khomenko, O.V. Yushchenko, Phys. Rev. E 68 (2003) 036110.

[6] A.V. Khomenko, Phys. Lett. A 329 (1-2) (2004) 140.

[7] A.V. Khomenko, I.A. Lyashenko, Technical Phys. 50 (11) (2005) 1408.

[8] A.V. Khomenko, I.A. Lyashenko, Cond. Matt. Phys. 9 (4(48)) (2006) 695.

[9] G. Reiter, A.L. Demirel, J. Peanasky, L.L. Cai, S. Granick, J. Chem. Phys. 101 (3) (1994) 2606.

[10] A.L. Demirel, S. Granik, J. Chem. Phys. 109 (16) (1998) 6889.

[11] A.I. Olemskoi, A.V. Khomenko, Zh. Eksp. Teor. Fiz. 110 (6(12)) (1996) 2144;

A.I. Olemskoi, A.V. Khomenko, JETP 83 (6) (1996) 1180.

[12] F.R. Eirich (Ed.), Rheology: Theory and Applications, Academic Press, New York, 1960, p. 354.

[13] H. Haken, Synergetics. An Introduction. Nonequilibrium Phase Transitions and Self-organization in Physics, Chemistry, and Biology, third ed., Springer, Berlin, 1983, p. 371. 
[14] G. Luengo, J. Israelachvili, S. Granick, Wear 200 (1996) 328.

[15] V.L. Popov, Technical Phys. 46 (5) (2001) 605.

[16] G. Reiter, A.L. Demirel, J. Peanasky, L. Cai, S. Granick, The solid-like state of a confined liquid lubricant: Deformation and time effects, in: B.N.J. Persson, E. Tosatti (Eds.), Physics of Sliding Friction, Kluwer, Dordrecht, 1995, pp. 119-138.
[17] E.A. Brener, V.I. Marchenko, JETP Lett. 76 (4) (2002) 211.

[18] O.M. Braun, A.G. Naumovets, Surf. Sci. Rep. 60 (2006) 79.

[19] L.B. Zuev, V.I. Danilov, Philos. Mag. A 79 (1) (1999) 43. 